\title{
LA GEO-ALFABETIZACIÓN SOBRE LA DIVISIÓN TERRITORIAL ESPAÑOLA DE LOS FUTUROS MAESTROS DE EDUCACIÓN PRIMARIA EN LA UNIVERSIDAD DE SALAMANCA
}

\author{
Alejandro Gómez-Gonçalves \\ Universidad de Salamanca \\ Escuela Universitaria de Magisterio de Zamora. Campus Viriato \\ algomez@usal.es \\ ORCID iD: https://orcid.org/0000-0002-4988-4623 \\ Jaume Binimelis Sebastián \\ Universitat de les Illes Balears \\ jaume.binimelis@uib.es \\ ORCID iD: https://orcid.org/0000-0002-5256-0371 \\ Juan Antonio García-González \\ Universidad de Castilla-La Mancha \\ juanantonio.garcia@uclm.es \\ ORCID iD: https://orcid.org/0000-0001-7049-1085 \\ Isabel María Gómez-Trigueros \\ Universidad de Alicante \\ isabel.gomez@ua.es \\ ORCID iD: https://orcid.org/0000-0003-4666-5035
}

\section{Recibido: 27/03/2020; Aceptado: 12/04/2021; Publicado: 17/06/2021}

Cómo citar este artículo/citation: Gómez-Gonçalves, Alejandro; Binimelis Sebastián, Jaume; García-González, Juan Antonio y Gómez-Trigueros, Isabel María (2021). La geo-alfabetización sobre la división territorial española de los futuros maestros de Educación Primaria en la Universidad de Salamanca. Estudios Geográfıcos, 82 (290), e067. https://doi.org/10.3989/ estgeogr.202079.079

RESUMEN: Existe un creciente interés por medir el conocimiento previo y la adquisición de competencias y contenidos por parte del alumnado y del futuro profesorado, algo que, en los últimos años, también se ha extendido al ámbito de la geografía. El objetivo de esta investigación es analizar la alfabetización en geografía de una muestra de estudiantes universitarios del grado en Maestro de Educación Primaria por su posible efecto multiplicador, ya que serán ellos quienes enseñen geografía a las generaciones futuras. Para alcanzar el objetivo formulado se contó con la participación de 168 estudiantes de la Universidad de Salamanca, que respondieron un cuestionario compuesto por preguntas de carácter sociodemográfico y por una pregunta en la que debían dibujar un mapa mental sobre la división político-administrativa de España. Los resultados indican que los encuestados tienen ciertas dificultades para situar las provincias de nuestro país, especialmente aquellas más alejadas de su lugar de procedencia.

PALABRAS CLAVE: alfabetización geográfica, mapa mental, educación superior, estudiantes universitarios, organización territorial de España.

\section{ANALYSIS AND EVALUATION OF THE KNOWLEDGE ABOUT THE SPANISH TERRITORIAL DIVISION OF PRE- \\ SERVICE PRIMARY EDUCATION TEACHERS. REFLECTIONS IN RELATION TO GEO-LITERACY OF UNDER- \\ GRADUATE STUDENTS AT THE UNIVERSITY OF SALAMANCA}

ABSTRACT: Today there is a growing interest in measuring the academic performance and the acquisition of certain skills and content by students at different ages. It has also been extended to the field of geography in recent years. The objective of this paper is to analyse the geo-literacy of a sample of university students of the degree in Primary Education Teacher by its possible multiplier effect, since they will be the ones who teach geography to future generations. In order to achieve the formulated objective, 168 students from the University of Salamanca participated in this research. They were administered a questionnaire made up of socio-demographic questions and a question in which the respondents have to draw a mental map of the political-administrative division of Spain. The results indicate that those surveyed students have some difficulties in locating the provinces of our country, especially those furthest from their place of origin.

KEY WORDS: geo-literacy, mental map, higher education, university students, Spanish territorial organization. 


\section{INTRODUCCIÓN}

Entre los países de nuestro entorno existe una preocupación creciente por medir el rendimiento académico del alumnado y la adquisición de determinadas competencias y contenidos. Uno de los instrumentos más conocido es el informe PISA (por sus siglas en inglés: Programme for International Student Assessment o informe del Programa Internacional para la Evaluación de Estudiantes), que examina a estudiantes de 15 años sobre lectura, matemáticas y ciencias naturales, además de analizar otros temas relacionados con la educación y la pedagogía docente. En concreto, el informe de 2018 subrayaba la importancia de las competencias y los conocimientos en ciencias, ya que se asume que las personas con conocimientos científicos serán capaces de tomar decisiones más informadas que aquellas que no los tienen (OECD, 2019).

En ámbitos académicos, la definición de alfabetización científica (science literacy o scientific literacy, que es la versión empleada en el informe PISA) es un tema de gran actualidad y no exento de debate (Roberts y Bybee, 2014). En las últimas décadas, han sido muy numerosas las investigaciones sobre los contenidos en ciencias naturales impartidos en las escuelas y sobre la formación de la ciudadanía en materias de ciencias, especialmente dentro de la literatura anglosajona (Cavagnetto, 2010; Rudolph y Horibe, 2015). En España, el volumen de trabajos sobre este tema es sensiblemente menor, aunque, como recientemente se han publicado varios estudios sobre alfabetización científica (Archila, Molina y Truscott de Mejía, 2019; Díaz Moreno, 2019). Sin embargo, hay un fuerte contraste entre el gran interés que aparentemente existe en el ámbito de las ciencias naturales por conocer cómo se enseñan estas disciplinas y qué saben los estudiantes y los ciudadanos sobre ellas, y el menor número de investigaciones con el mismo objetivo desarrolladas en el entorno de las ciencias sociales y, más concretamente, en el ámbito de la geografía.

La inquietud por conocer el grado de alfabetización geográfica de ciudadanos y estudiantes arrancó en Estados Unidos en la década de los ochenta del siglo pasado. Se considera que el estudio de Saarinen (1987) fue el primero que analizó la alfabetización geográfica (geographic literacy o geo-literacy) tomando como muestra a un conjunto de más de 3.500 estudiantes universitarios de más de cincuenta países y de 75 universidades distintas, marcando un hito en dicha temática. En los últimos años, hemos asistido a un repunte de los estudios sobre geo-alfabetización en países tan dispares como República Checa (Polons- ky, Novotny y Lysak, 2010), Hungría (Rédei, Kincses y Jakobi, 2011), Turquía (Sudas y Gokten, 2012), Estados Unidos (Nishimoto, 2012), Chile (Badillo, Crespo y Hass, 2017) o Indonesia (Ikhsan, Kurniato, Apriyanto y Nurdin, 2018), por citar solo algunos ejemplos. En España se han publicado recientemente algunas investigaciones sobre conocimientos mínimos o alfabetización geográfica (Binimelis y Ordinas, 2016; Binimelis y Ordinas, 2018; Binimelis et al., 2018; Rubio y Martínez, 2014) que comparten el hecho de que la muestra la forman estudiantes del Grado de Maestro en Educación Primaria por su potencial efecto multiplicador. Es decir, se indaga sobre los conocimientos mínimos en geografía de los futuros maestros, puesto que ellos serán los responsables de enseñar geografía a las próximas generaciones.

Turner y Leydon (2012) definen la geo-alfabetización como la habilidad para entender, procesar y utilizar la información espacial, y consideran que involucra dos habilidades distintas pero complementarias: a) el conocimiento geográfico (memorización de nombres de lugar y atributos); b) identificación geo-espacial (localización de lugares y atributos en un mapa). Sin embargo, la geo-alfabetización también comprende niveles más complejos, tal y como señala Memisoglu (2017), quien establece tres niveles en función de su complejidad: un nivel elemental, en el que se emplean herramientas para fijar el conocimiento de los nombres de lugar y su localización; un nivel intermedio, en el que se incluyen las relaciones entre el hombre y el medio y las causas implícitas en ellas; y un nivel más alto, que exige a los estudiantes tener herramientas de comprensión crítica y habilidades para entender las relaciones de la geografía con los recursos naturales y las dificultades de las naciones. Por tanto, el conocimiento de los nombres de lugar y su localización se encontraría en el lugar más bajo de la geo-alfabetización, aunque conviene señalar que la localización es uno de los cuatros pilares básicos de la ciencia geográfica y tiene un peso decisivo en otros dos (Gershmel, 2005): 1) localización: saber dónde están las cosas; 2) lugar: entender el carácter único de un lugar y las diferencias con otros lugares; 3) conexiones: establecer vínculos entre diferentes localizaciones; 4) regiones: manejar patrones espaciales en una escala más amplia. Conviene señalar que el conocimiento geográfico ayuda a construir estructuras esquemáticas en la mente que funcionan a largo plazo y permiten procesar, por ejemplo, la información sobre lugares que aparecen en artículos periodísticos o libros (Nolan, 2002). 
La preocupación que subyace en buena parte de la literatura científica que aborda el tema de la geo-alfabetización es saber si los estudiantes o los ciudadanos poseen los conocimientos mínimos para comprender el mundo global en el que les ha tocado vivir, donde los fenómenos ocurren en territorios diversos y las personas debemos establecer una conexión espacial para entender dónde y por qué se producen esos fenómenos (Nishimoto, 2012). Así:

"la asignatura de geografía ofrece un vasto campo de concreción de las competencias de ciudadanía, especialmente las que implican el desarrollo de la identidad cultural y territorial, así como la reflexión y participación crítica sobre los problemas que a diversas escalas afectan al mundo en que vivimos" (Cachinho y Reis, 2011; p. 190).

De este modo, la formación geográfica se hace necesaria para sensibilizar a la ciudadanía sobre el territorio en el que habita y sobre su propio país, contribuyendo a fomentar la identidad de la comunidad (Santiago, 2017). A este respecto es necesario apuntar que, históricamente, la enseñanza de la geografía ha contenido una fuerte carga ideológica (Claudino, 2010), ya que una de sus características principales era el refuerzo de la identidad territorial y la consolidación del espíritu nacional (Souto, 2008). En el caso español, parece evidente que, si se intentan entender las raíces de algunos de los principales problemas del país durante el siglo XXI, tales como las tensiones territoriales o el fenómeno de la despoblación, por citar solo dos ejemplos, se ha de tomar como punto básico de partida la localización territorial de estos fenómenos.

En la Declaración Internacional sobre Educación Geográfica de 2016, la Unión Geográfica Internacional (UGI) reafirmó el papel de la disciplina geográfica para la vida de los ciudadanos en un mundo fuertemente interconectado, señalando algunas preguntas esenciales que deben incluir las investigaciones sobre educación geográfica (Kolossov, 2016), tales como ¿qué geografía necesitan conocer los estudiantes? y ¿cuáles son sus precepciones erróneas y concepciones previas? Además, algunos autores apuntan que la evaluación de la alfabetización geográfica debería incorporar la identificación espacial como punto de partida (Bascom, 2011), algo que se puede lograr empleando el mapa mental como instrumento.

\section{La utilidad de los mapas mentales para medir la geo-} alfabetización

Los estudios sobre alfabetización geográfica se han realizado, habitualmente, a través de exámenes escritos, de pruebas tipo test o a través de mapas mudos (Álvarez y Córdoba, 2015). Sin embargo, en esta investigación se empleará el mapa mental como instrumento para la recogida de información. Desde la óptica de la Geografía de la percepción, algunos autores han ido empleado en sus investigaciones los mapas que, a modo de bocetos, habían elaborado estudiantes de universidades repartidas por los cinco continentes (Saarinen y MacCabe, 1995) o por distintos municipios (Morales, 2015; Morales, Lázaro y Gómez, 2018, 2018). Este instrumento, el mapa mental o cognitive map, ha sido definido en la segunda edición de la International Encyclopedia of Human Geography como (Gold, 2009; p. 289): "Ios procesos mediante los cuales las personas adquieren, almacenan y recuerdan información de los lugares en los entornos con los que entran en contacto".

Según De Castro (1999) los mapas mentales pueden definirse como construcciones mentales que se sustentan a partir de ideas previas, conocimientos, experiencias y creencias. Las ventajas que plantea la utilización de los mapas mentales es que ofrecen información sobre la visión espacial de una persona (Nishimoto, 2012) y por ello son numerosos los estudios que los han utilizado para evaluar el conocimiento del mundo que tienen los estudiantes (Bascom, 2011; Polansky y Novotny, 2010). Este instrumento, se ha empleado en tres grandes ámbitos de investigación (Gold, 2009): a) la construcción y organización de los mapas mentales, examinando la manera en que esos mapas adquieren estabilidad e incorporan cambios a medida que se aprenden cosas nuevas, algo especialmente llamativo durante el periodo de crecimiento de los niños; b) el conocimiento de entornos urbanos, que es el ámbito al que se circunscribe el clásico trabajo de Kevin Lynch "La imagen de la ciudad" (1960); c) investigaciones de menor escala en las que se analizan imágenes regionales y mundiales.

Sin embargo, la utilización del mapa mental como instrumento para la obtención de datos empíricos presenta una serie de limitaciones (García, 2018): a) la metodología empleada para obtener la muestra suele diferir entre un estudio y otro, por lo que son pruebas difícilmente comparables entre sí; b) la información obtenida es difícil de sistematizar, lo que dificulta la realización de extrapolaciones de datos y las comparaciones espaciales o temporales entre distintos mapas. Por consiguiente, el mapa mental es fácil de conseguir, pero es difícil trabajar con él porque nos 
ofrece una gran cantidad de información y su tratamiento requiere de un trabajo arduo y casi artesanal. Aun así, son numerosos los trabajos que en nuestro país han empleado el mapa mental como instrumento metodológico durante los últimos años (Binimelis y Ordinas, 2018; García, 2018; Solís y Ureña, 2019) al permitir analizar el nivel de desarrollo del conocimiento geográfico de los individuos.

\section{El mapa de las provincias en el sistema educativo es- pañol}

Para trabajar sobre alfabetización geográfica a través de mapas mentales, se ha optado por analizar el grado de conocimiento de un conjunto de futuros maestros sobre el mapa de las provincias españolas. Este mapa ha estado siempre presente en el sistema educativo español: era el recurso didáctico más utilizado para la enseñanza de las ciencias sociales en las escuelas, tanto antes como después de la Constitución de 1978 (Corrochano, Gómez-Gonçalves, Sánchez-Barbero y Martín-Pastor, 2019), al permitir no solo trabajar la educación memorística a través de la enumeración de accidentes geográficos, ríos, provincias y sus principales manufacturas, sino al fomentar el estudio de las relaciones y pautas espaciales (Gersmehl y Gersmehl, 2011; Lee y Bednarz, 2012).

Desde el punto de vista del currículo escolar, y tomando como referencia Castilla y León, que es la comunidad autónoma donde se ha llevado a cabo esta investigación, las provincias aparecen por primera vez en la asignatura de Ciencias Sociales en el tercer curso de Educación Primaria. Es decir, con 8 años, todos los ciudadanos escolarizados en este territorio se enfrentan por primera vez al mapa provincial (DECRETO 26/2016, de 21 de julio). $Y$ en cuarto curso, "localiza en un mapa las distintas comunidades autónomas y ciudades autónomas que forman España, así como sus provincias" aparece como uno de los estándares de aprendizaje evaluables. El mapa provincial se empieza a estudiar en Educación Primaria y se retoma, de manera intensiva, en la asignatura de Ciencias Sociales del tercer curso de Educación Secundaria Obligatoria (ESO), especialmente en todo lo que atañe al estudio de la organización territorial de España (ORDEN EDU/362/2015, de 4 de mayo). Por su parte, en Historia de España, asignatura obligatoria de segundo curso de Bachillerato, el mapa provincial tiene un peso muy importante. Y, a su vez, en la asignatura de Geografía, optativa del itinerario de ciencias sociales, el mapa provincial juega un papel clave.

En resumen, cualquier persona escolarizada en esta comunidad autónoma ha estudiado el mapa provincial en la educación obligatoria desde los 8 años hasta finalizar esta etapa educativa, con 16 años. Sin embargo, Martín, Lozano y Lázaro (2006) llevaron a cabo un estudio sobre el conocimiento de las provincias españolas entre escolares donde evidenciaron un desconocimiento geográfico notable. Por ello, la presente investigación trata de continuar indagando en esa dirección, pero avanzando un paso más, es decir, analizando el manejo que tienen los estudiantes universitarios del mapa provincial. En concreto se ha optado por desarrollar este estudio con alumnos del grado de Maestro en Educación Primaria, ya que serán ellos los encargados de enseñar esta materia a las futuras generaciones.

\section{La utilidad del mapa provincial para medir la alfabe- tización geográfica}

La división territorial en unidades administrativas es a la vez que un sistema de gobernanza territorial, una abstracción de la que nos servimos para comprender mejor el territorio. La región, sea del tipo que sea, es una construcción mental del geógrafo que nos ayuda a entender y organizar el territorio (Puyol, 1980). Entre las múltiples tipologías de regiones, las cuales han ido evolucionando a lo largo de los vaivenes epistemológicos del pensamiento geográfico, las divisiones generadas por la estructura político-administrativa son algunas de las más utilizadas para estudios e investigaciones. Se han convertido en un tamiz de recogida y organización de información estadística que, desde el punto de vista del proceso de enseñanza-aprendizaje, suponen el marco en el que los estudiantes aprenden de manera compartimentada el medio geográfico. Las estructuras administrativas van desde una escala planetaria con países y unidades supranacionales, como puede ser la Unión Europea, a niveles de escala local con unidades inframunicipales. Todas ellas cuentan con una superficie y se agrupan quedando incluidas de forma unívoca en una estructura de rango superior.

En nuestro país, el Estado organiza su estructura territorial y de gestión en tres grandes niveles: municipio, provincia y comunidades autónomas. 
En concreto, el mapa provincial de España tiene una presencia explícita en el currículo escolar porque la provincia sigue siendo una unidad clave de la organización político-territorial de nuestro país, algo que queda recogido en el artículo 141 de la Constitución de 1978, y son las piezas básicas que componen las comunidades autónomas (artículos del 143 al 159). Además, todos los municipios de España se encuentran integrados en una y solo una provincia. De igual forma todas ellas pertenecen a una única comunidad autónoma, contando con continuidad espacial salvo las islas y algunos enclaves históricos. Por otra parte, todo este entramado territorial ha quedado integrado en la estructura administrativa europea, denominada Nomenclatura de las Unidades Territoriales Estadísticas (NUTS, por sus siglas en francés), que está compuesta por tres niveles de desagregación con los cuales se obtienen y comparan datos estadísticos de manera homogénea en la Unión Europea. Este mapa provincial de Javier de Burgos ha permanecido inalterado desde 1833 , con las excepciones de la creación de las dos provincias canarias en 1927 y de las ciudades autónomas de Ceuta y Melilla en 1995, dando como resultado un mapa bien definido y compuesto por 50 unidades, más las dos citadas ciudades autónomas. Por todo ello Burgueño (2011) señala que:

"podríamos decir que historiar las provincias es referirse a un elemento familiar y arraigado en el imaginario colectivo de múltiples generaciones. En España ha habido guerras civiles, se han sucedido monarquía y república, dictadura y democracia, centralización y autonomía... y entre tanto, la división provincial ha persistido incólume (p. 9)".

Por tanto, sería lógico esperar que un alumno universitario que ha cursado la enseñanza obligatoria y el bachillerato en nuestro país, tuviera una noción bastante acertada del mapa provincial.

Por todo ello, los objetivos que planteamos en esta investigación son los siguientes: 1) analizar los conocimientos sobre la organización territorial de España de un grupo de estudiantes del grado de Maestro en Educación Primaria; 2) examinar si existen diferencias significativas entre género, edad, origen, formación o profesión de los padres a la hora de conocer el mapa de las provincias españolas; 3) valorar la consistencia del mapa mental como instrumento con el cual desarrollar esta prueba.

\section{METODOLOGÍA}

La metodología incluye la justificación y elaboración del protocolo de recogida de información, su tabulado y su análisis y presentación final. Podemos estructurarla en tres pilares: muestra, instrumento y procedimiento.

\section{Muestra}

Los participantes en este estudio son estudiantes universitarios que estaban cursando el segundo curso del grado de Maestro de Educación Primaria, que en la Universidad de Salamanca se imparte en tres centros distintos. Se contó con la participación voluntaria de 167 estudiantes que constituyen la muestra de este estudio (ver Tabla 1). La mayoría son mujeres, más de dos tercios de la muestra, con una media de edad de 20,5 años. En lo referente al centro universitario, un $32,3 \%$ cursaba sus estudios en la Escuela Universitaria de Magisterio de Zamora, un 32,3\% en la Escuela Universitaria de Educación y Turismo de Ávila y un $35,3 \%$ en la Facultad de Educación de Salamanca. La ligera diferencia en el número de encuestados entre los tres centros a la hora de recoger la muestra se debe a la diferencia de matrícula entre ellos. La mayoría de los estudiantes procede de las tres provincias en las que están situados dichos centros universitarios, siendo destacable también la presencia de alumnos originarios de Cáceres $(7,2 \%)$, de León $(3,6 \%)$ y de Valladolid (2,4\%).

La formación de los progenitores muestra una distribución equilibrada entre los dos géneros, identificándose un nivel de formación más elevado entre las madres. En general, predominan los padres y madres con estudios mínimos, seguidos, en el caso de los padres, de aquellos que tienen estudios secundarios, mientras que, en el caso de las madres, en segundo lugar, aparecen aquellas que tienen estudios superiores. Es necesario subrayar que, pese a que las mujeres presentan una mayor formación (el $24 \%$ cuenta con estudios superiores frente al $16,8 \%$ en el caso de los padres, y únicamente el $25,7 \%$ posee estudios mínimos frente al $33,5 \%$ en el caso de los padres), existe un amplio colectivo que asume las tareas domésticas (18,6\% de amas de casa) frente a un porcentaje ínfimo de varones que asumen ese rol. Por otra parte, si se analizan las profesiones, se observa un predominio del sector terciario (cualificado o sin cualificación), destacando, en el caso de las ma- 
TABLA 1

CARACTERÍSTICAS DE DISTRIBUCIÓN DE LA MUESTRA ( $\mathrm{N}=167)$

\begin{tabular}{|c|c|c|}
\hline & $\mathbf{n}$ & $\%$ \\
\hline \multicolumn{3}{|l|}{ Género } \\
\hline Masculino & 54 & 32,3 \\
\hline Femenino & 112 & 67,1 \\
\hline \multicolumn{3}{|l|}{ Provincia de origen } \\
\hline Ávila & 44 & 26,3 \\
\hline Salamanca & 30 & 18,0 \\
\hline Zamora & 35 & 21,0 \\
\hline Cáceres & 12 & 7,2 \\
\hline León & 6 & 3,6 \\
\hline Valladolid & 4 & 2,4 \\
\hline Otras provincias & 36 & 21,6 \\
\hline \multicolumn{3}{|l|}{ Estudios del padre } \\
\hline Sin estudios & 7 & 4,2 \\
\hline Básicos & 49 & 29,3 \\
\hline ESO, EGB, Bachillerato Elemental (o estudios secundarios) & 33 & 19,8 \\
\hline Bachillerato superior & 21 & 12,6 \\
\hline FP & 14 & 8,4 \\
\hline Estudios superiores & 28 & 16,8 \\
\hline Doctorado & 0 & 0,0 \\
\hline \multicolumn{3}{|l|}{ Profesión del padre } \\
\hline Paro & 2 & 1,2 \\
\hline Sector primario & 13 & 7,8 \\
\hline Sector secundario & 0 & 0,0 \\
\hline Sector terciario sin cualificación & 101 & 60,5 \\
\hline Sector terciario cualificado & 32 & 19,2 \\
\hline Ama/o de casa & 1 & 0,6 \\
\hline Jubilado/a & 7 & 4,2 \\
\hline
\end{tabular}

Elaboración propia.

dres, el ya mencionado porcentaje que trabaja de ama de casa $(18,6 \%)$.

\section{Instrumento}

Para medir la geo-alfabetización los participantes han rellenado de manera voluntaria un cuestionario sobre datos socio-demográficos y han realizado un mapa mental sobre la división político-administrativa de España, siguiendo el modelo empleado por Binimelis y Ordinas (2018) para el estudio de caso de las Islas Baleares.

A los encuestados se les entregó una hoja tama-

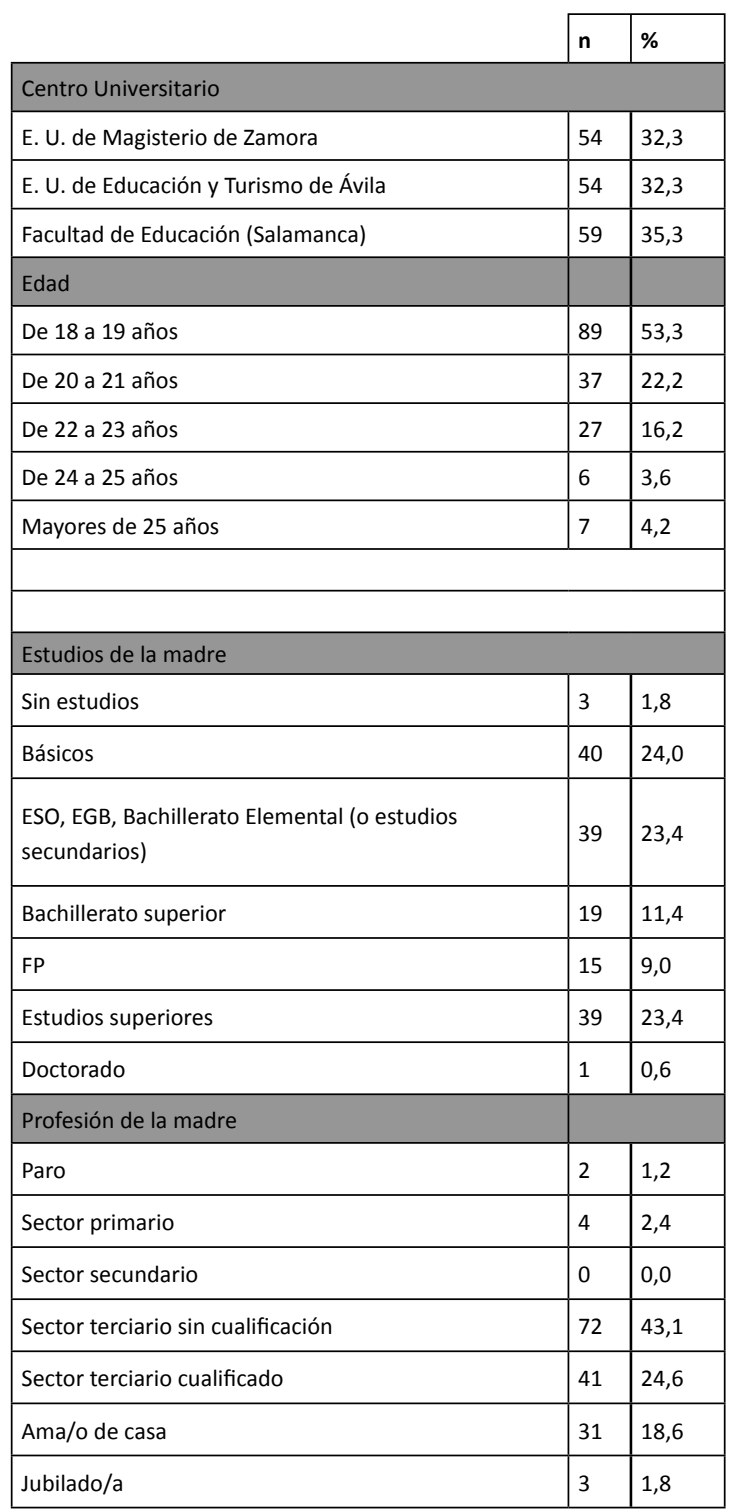

ño DIN A4 para la realización del mapa mental y contaron con treinta minutos para su realización. Dicha prueba se llevó a cabo durante los primeros días del curso académico 2018-2019, entre alumnos del segundo curso del grado en Maestro en Educación Primaria, y antes de haber cursado alguna asignatura relacionada con las ciencias sociales. Además, los participantes no recibieron ningún tipo de ayuda ni ninguna indicación sobre leyenda, puntos cardinales o escala y cada encuestado firmó una carta de consentimiento para el buen uso de la información y de los datos personales adjuntados con finalidades científicas. 
El análisis de la información recopilada en el cuestionario socio-demográfico fue clasificada en un sistema de categorías que permitió agrupar el nivel de formación y la profesión de los progenitores. Tras establecer dichas categorías, cuatro investigadores externos validaron dicho protocolo de recogida de información y su posterior categorización. En cuanto a la información obtenida de los mapas mentales, en esta investigación se ha analizado la presencia de las unidades provinciales en el mapa de la división político-administrativa de España. La valoración ordinal escogida es una sencilla escala de Likert con tres valores $(0,1$ y 2$)$. La elección de uno u otro se ha hecho en función de la aparición o no de tres criterios: rotulación, localización y dibujo del elemento cartográfico. La utilización de etiquetas (topónimos o nombres de lugar) en un mapa mental facilita tanto la elaboración de la prueba a los encuestados como también, la tabulación de resultados por parte de los investigadores. Sin embargo, la utilización de mapas mentales como fuente de información induce a una mayor profundidad en el análisis frente a la simple rotulación de nombres de lugar en mapas mudos (lo que se denomina place, location, knowledge). La rotulación del topónimo del elemento es el más fácil de reconocer valorándolo positivamente independientemente de dónde esté situado, mientras que la localización y el dibujo son variables cuya evaluación es compleja. Es necesario destacar que en esta investigación no se ha atendido a la corrección ortográfica de los topónimos.

El valor más elevado se otorgaba cuando se aprecia un inequívoco conocimiento del elemento representado, lo que suponía que cumpliera correctamente con los tres criterios. En el extremo contrario aparece una valoración de 0 cuando el estudiante omite el elemento a valorar. La puntuación de 1 implica que el encuestado debe mostrar cierto conocimiento del enclave sin llegar a una precisión sobre este, lo que implica que debe cumplir con alguno de los anteriores criterios.

Conviene señalar que la fiabilidad entre codificadores se realizó de manera iterativa. Se escogieron 5 mapas de cada uno de los campus y fueron valorados por todos los codificadores. Todos los codificadores evaluaron los mismos mapas y fueron revisadas una a una todas las valoraciones. Este proceso fue repetido hasta tres veces lo que provocó que se afinaran y homogeneizaran los criterios de asignación. Tanto con el valor 2 como con el valor 0 no hubo ningún problema desde el inicio. Las discrepancias surgieron en la asignación del valor 1 cuya casuística llevo a que se rechazara el análisis de este valor. Por tanto, en esta investigación solo se tuvieron en cuenta los aciertos, es decir, las provincias o ciudades autónomas que recibieron una valoración de 2 en la escala de Likert, ya que en ellas los estudiantes demostraron un inequívoco conocimiento del elemento representado.

\section{Procedimiento}

El proceso metodológico comenzó con una búsqueda bibliográfica apoyada en trabajos previos, justificando por qué, a día de hoy, son numerosos los autores que siguen empleando el mapa mental para evaluar la geo-alfabetización. A partir de ahí, se contactó con la población objeto de estudio y se le invitó a que participara de manera voluntaria en esta investigación, garantizando su anonimato y el uso de los resultados con fines investigadores, a través de un cuestionario en el que se incluía una pregunta en la que los encuestados debían dibujar un mapa políticoadministrativo de España de forma presencial y en papel. Posteriormente se analizaron los datos obtenidos de los mapas mentales atendiendo a la correcta localización de las provincias y las ciudades autónomas. Para los análisis cuantitativos se realizó, por un lado, el estadístico de Kolmorov-Smirnov para identificar el comportamiento normal de la variable y se realizaron pruebas no paramétricas para relacionar esta variable con algunos indicadores categóricos (Clemente, 1992).

\section{RESULTADOS}

A partir del análisis de los mapas mentales de la organización territorial de España realizados por los 167 estudiantes del Grado en Maestro en Educación Primaria de la Universidad de Salamanca, se observa un mejorable conocimiento del mapa provincial de nuestro país. En cada uno de los mapas mentales se analizó la corrección en la ubicación de provincias y ciudades autónomas, bien rotuladas y bien dibujadas con un polígono cerrado, como ya se indicó en la metodología. Los resultados muestran que sobre un total de 52 puntos posibles ( 50 provincias y 2 ciudades autónomas), el conjunto de los encuestados ob- 
tuvo una puntuación promedio de 21 puntos, lo que pone de manifiesto la ausencia de un conocimiento riguroso del mapa provincial. En la Tabla 2 se pueden observar las puntuaciones obtenidas por el conjunto de los estudiantes encuestados, donde destaca sobremanera que el $58,4 \%$ del total de los futuros maestros obtuvo menos de 25 puntos, por tanto, no fueron capaces de ubicar de manera correcta ni siquiera la mitad de las provincias de nuestro país. Este resultado da lugar a profundas reflexiones sobre las debilidades del sistema educativo obligatorio y también del universitario. Es muy notable el hecho de que cerca de un tercio de los futuros maestros no fuera capaz de ubicar, al menos, 10 provincias o ciudades autónomas de manera correcta $(30,7 \%$ de los encuestados) y que, en el otro extremo, únicamente una alumna localizara correctamente todas las provincias. En este análisis de aciertos totales se observa una diferenciación muy marcada desde el punto de vista del género. En el caso de los hombres, la valoración promedio de sus mapas mentales fue de 17 puntos $(32,7 \%$ de aciertos), mientras que la puntuación promedio obtenida por las mujeres fue de 22,8 puntos $(43,8 \%)$, es decir, se observa una diferenciación de más de diez puntos porcentuales entre mujeres y hombres. Siendo esta una cuestión controvertida, las mujeres parecen obtener mejores notas en asignaturas de Geografía que los chicos, como se ha demostrado en varios estudios sobre esta materia (Butt, Weeden y Wood, 2004; Clark, Monk y Yool, 2007; Smith, 2004).
Si relacionamos la puntuación obtenida por el alumnado participante con algunas variables que forman parte de su entorno socio-económico, es posible identificar algunos hechos notables. En primer lugar, la distribución de valores a que da lugar la evaluación obtenida por el alumnado participante se comporta de forma asimétrica, al no amoldarse a la curva de distribución normal (prueba de Kolmorov-Smirnov con un nivel de significación $p<0,05)$. Esto se debe al peso que en su distribución tienen las evaluaciones bajas (alumnado con una puntuación muy baja), razón por la cual ha sido necesario emplear pruebas no paramétricas para relacionar esta variable con algunos indicadores de carácter categórico. Se ha identificado una relación entre el nivel educativo de los padres y madres (estudios mínimos, básicos, secundarios y superiores) y las puntuaciones obtenidas al rechazarse la hipótesis nula en la prueba no paramétrica de Kruskal-Wallis ( $p<0,05$ en ambos casos). Aunque las medias de los diferentes grupos no difieran mucho entre sí (ver Tabla 3), existe una relación entre ambas variables. En otros ámbitos científicos distintos, la influencia del nivel de estudios de los padres y el rendimiento académico de los alumnos es un tema que se ha analizado durante las últimas décadas, ya que desde el campo de la psicología se ha puesto de manifiesto la importancia que tienen las variables contextuales de los estudiantes a la hora de explicar su rendimiento académico. Dentro de este grupo de variables tienen un papel muy destacado las características de la

TABLA 2

PORCENTAJE DE ACIERTOS DE LA POBLACIÓN ENCUESTADA

\begin{tabular}{|c|c|c|c|c|c|c|}
\hline Aciertos total & $\%$ total & $\%$ total acumulado & $\%$ hombres & $\begin{array}{l}\% \text { hombres } \\
\text { acumulado }\end{array}$ & $\%$ mujeres & $\begin{array}{l}\% \text { mujeres } \\
\text { acumulado }\end{array}$ \\
\hline $0-4$ & 16,9 & 16,9 & 27,8 & 27,8 & 11,7 & 11,7 \\
\hline $5-9$ & 13,9 & 30,7 & 14,8 & 42,6 & 13,5 & 25,2 \\
\hline $10-14$ & 7,8 & 38,6 & 5,6 & 48,1 & 9,0 & 34,2 \\
\hline $15-19$ & 10,2 & 48,8 & 9,3 & 57,4 & 10,8 & 45,0 \\
\hline $20-24$ & 9,6 & 58,4 & 11,1 & 68,5 & 9,0 & 54,1 \\
\hline $25-29$ & 11,4 & 69,9 & 9,3 & 77,8 & 13,5 & 67,6 \\
\hline $30-34$ & 9,6 & 79,5 & 13,0 & 90,7 & 8,1 & 75,7 \\
\hline $35-39$ & 5,4 & 84,9 & 3,7 & 94,4 & 5,4 & 81,1 \\
\hline $40-44$ & 7,8 & 92,8 & 1,9 & 96,3 & 9,9 & 91,0 \\
\hline $45-49$ & 6,6 & 99,4 & 3,7 & 100,0 & 8,1 & 99,1 \\
\hline $50-52$ & 0,6 & 100,0 & 0,0 & & 0,9 & 100,0 \\
\hline
\end{tabular}

Elaboración propia. 
TABLA 3

PROMEDIO DE ACIERTOS EN FUNCIÓN DEL NIVEL DE ESTUDIOS Y LA PROFESIÓN DE LOS PADRES (\%)

\begin{tabular}{|c|c|c|c|c|c|c|c|}
\hline Estudios padre & $\begin{array}{l}\text { Promedio } \\
\text { aciertos }\end{array}$ & Estudios madre & $\begin{array}{c}\text { Promedio } \\
\text { aciertos }\end{array}$ & Profesión padre & $\begin{array}{l}\text { Promedio } \\
\text { aciertos }\end{array}$ & Profesión madre & $\begin{array}{c}\text { Promedio } \\
\text { aciertos }\end{array}$ \\
\hline Sin estudios & 20,9 & Sin estudios & 15,7 & Desemplead & 35,5 & Desemplead & 15,5 \\
\hline Básicos & 22,8 & Básicos & 23,7 & Sector primario & 28,5 & Sector primario & 22,8 \\
\hline Estudios secundarios & 14,1 & Estudios secundarios & 15,0 & Sector secundario & & Sector secundario & \\
\hline Bachillerato superior & 22,4 & Bachillerato superior & 19,0 & Sector terciario sin cuali. & 20,8 & Sector terciario sin cuali. & 20,4 \\
\hline FP & 22,8 & FP & 23,0 & Sector terciario cuali. & 21,4 & Sector terciario cuali. & 23,5 \\
\hline \multirow[t]{2}{*}{ Estudios superiores } & 22,5 & Estudios superiores & 23,4 & Ama/o de casa & 12,0 & Ama/o de casa & 20,4 \\
\hline & & Doctorado & 35,0 & Jubilado/a & 14,9 & Jubilado/a & 19,3 \\
\hline
\end{tabular}

Elaboración propia.

familia y, especialmente, el nivel de estudios de los padres (Garbanzo, 2007). Algunas investigaciones han demostrado altas correlaciones entre el nivel de estudios de los progenitores y el rendimiento académico de sus hijos (Hernando, Oliva y Pertegal, 2012) e, incluso, se incide en la consideración del nivel académico de la madre como una variable fundamental en el rendimiento académico ya que, en ocasiones, es ella la que se encarga de gestionar la educación de los hijos (Pérez y Castejón, 1998).

En cuanto a la representación cartográfica de los mapas mentales realizados por los estudiantes encuestados, se ha optado por mostrar una representación conjunta del mapa provincial de España, dándole un color a cada provincia en función del porcentaje de aciertos entre la población encuestada. Se ha optado por una gama cromática que va del amarillo hasta el marrón, apareciendo en colores más claros las provincias que han obtenido un menor número de aciertos y en colores oscuros las que más veces fueron ubicadas de manera correcta, es decir, las que aparecen de manera más nítida en el mapa mental de los encuestados. No se han valorado ni, por tanto, cartografiado, los espacios en blanco o terra incognita de los mapas mentales, así como tampoco la presencia o la ausencia de los países limítrofes, por lo que no se ha incorporado información sobre ellos.

En la Figura 1 se ha representado cartográficamente la información obtenida del análisis de los 167 mapas mentales que componen la muestra. En dicha imagen se observa que las provincias que aparecen en colores más oscuros, es decir, las que han sido identificadas de manera correcta en un mayor número de veces, son Madrid, identificada de manera correcta por el 85,6\% de los encuestados y de donde eran originarios únicamente el $1,8 \%$ de los estudiantes analizados, y Asturias $(80,2 \%)$, seguidas de Murcia $(75,4 \%)$, Cantabria $(68,3 \%)$, Salamanca $(64,7 \%)$, de donde eran originarios el $18 \%$ de los encuestados y en cuya Universidad estudiaba la totalidad de la población muestreada, las Islas Baleares (62,3 $\%)$, Badajoz y León, ambas con un porcentaje del 60,5 . En sentido contrario, las provincias menos conocidas fueron las dos canarias, dibujadas de manera correcta únicamente por el 7,2\% de los encuestados, las tres vascas, de las cuales Bizkaia fue ubicada de manera correcta por el $12 \%$ de los encuestados, mientras que Gipuzkoa y Álava por $13,2 \%$, y Girona con el $19,8 \%$. Son seis territorios alejados de los lugares de origen mayoritarios de los encuestados, pertenecientes a tres comunidades autónomas con cierta personalidad histórica y que, sin embargo, las provincias que las integran parecen ser desconocidas para los estudiantes que componen la muestra analizada. Estos datos se asemejan a los obtenidos por Martín, Lozano y Lázaro (2006) en su trabajo con estudiantes madrileños, ya que las provincias menos conocidas también fueron las dos canarias y las tres vascas.

Si se realiza un análisis en conjunto de las provincias que componen la Figura 1 se observa que el territorio mejor conocido coincide, a grandes rasgos, con el entorno más próximo de las provincias de origen mayoritario de los alumnos encuestados (Ávila, Salamanca, Zamora, Cáceres, León): Madrid, Asturias, Cantabria, las provincias de Castilla y León y de Extremadura, La Rioja, son los territorios mejor conocidos por los encuestados y todas ellas están situadas dentro 


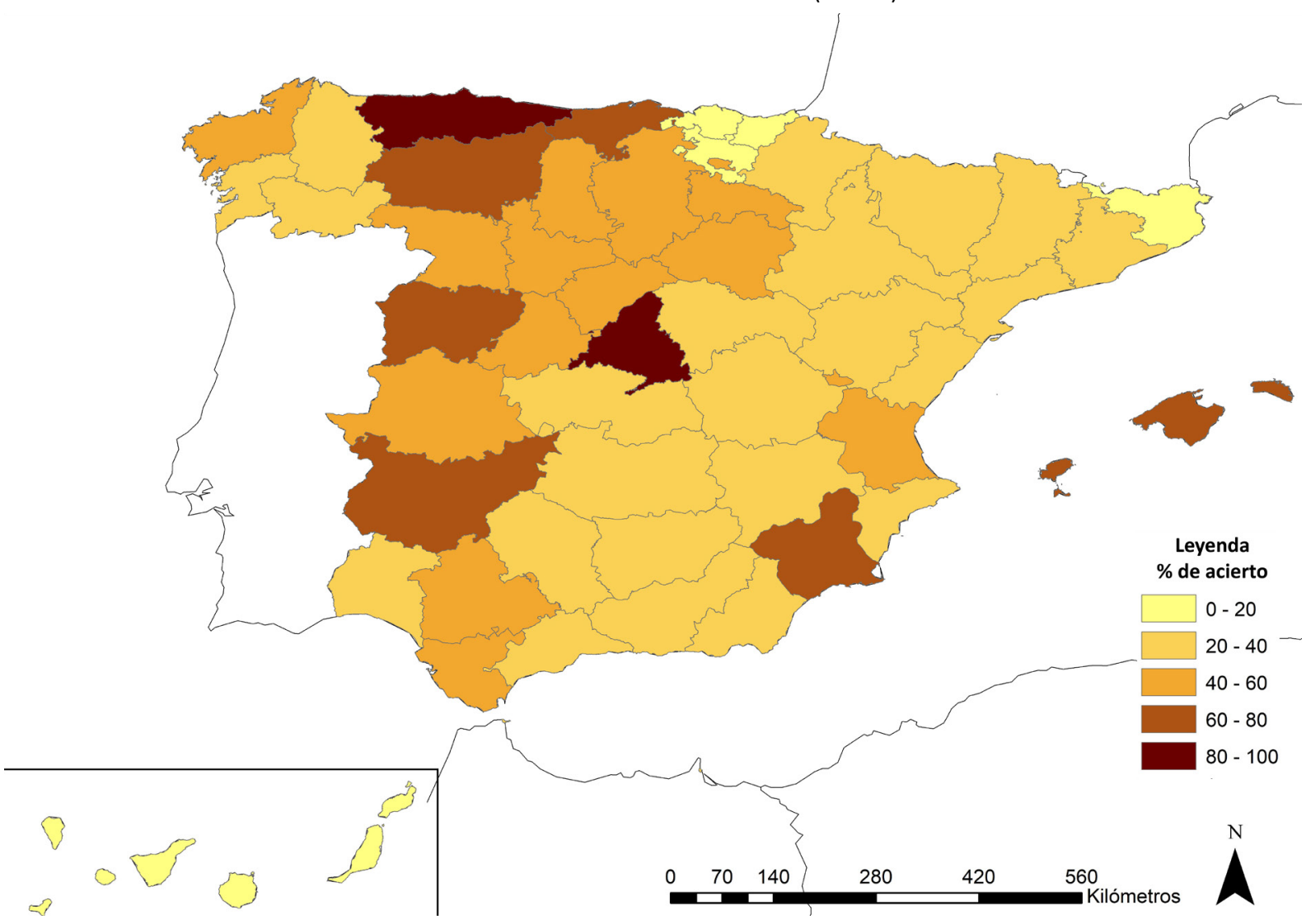

del primer y del segundo cuartil del ranking de provincias mejor identificadas (ver Tabla 4). Pese a estar situadas dentro del segundo cuartil, las provincias gallegas, con la excepción de Pontevedra, tienen porcentajes de acierto relativamente bajos: A Coruña es la provincia con mayor porcentaje de aciertos $(43,7 \%)$, mientras que Pontevedra solo fue ubicada bien por el $36,5 \%$ de los encuestados. El resto del país, en una media luna que comienza en el País Vasco y que recoge la mitad oriental de la península incluyendo también las provincias andaluzas y las canarias, es un territorio poco conocido para los alumnos encuestados, salvo alguna excepción puntual: Baleares, además de la ya citada provincia de Murcia, suponen un caso singular dentro de esta media luna oriental compuesta por 35 provincias que son poco conocidas por los futuros maestros de la Universidad de Salamanca, junto con Valencia, Sevilla y Cádiz.
Las tres principales provincias de origen de los estudiantes encuestados fueron aquellas en las que se encontraban los centros universitarios donde se distribuyó el cuestionario. Son provincias de Castilla y León muy próximas entre sí, por lo que cabría esperar que los mapas mentales fueran también bastante semejantes. Al tratar de buscar explicaciones a los resultados desde la consideración del lugar de residencia o la provincia de procedencia, se ha atendido al grupo de mapas cuyos autores proceden de Cáceres (ver Figura 2). Al analizar dicha figura se observa, a primera vista, que el índice de respuestas correctas es más elevado en el caso de los estudiantes originarios de la provincia de Ávila: el promedio de acierto por provincia de los abulenses $(n=44)$ fue de un $56,6 \%$, mientras que en el caso de los cacereños esta cifra fue del $48,7 \%(n=12)$, del $36 \%$ en el caso de los salmantinos ( $n=30$ ) y $27,4 \%$ en el de los zamoranos $(n=35)$. Las provincias que aparecen dibujadas de manera correcta en 
TABLA 4

CLASIFICACIÓN DE LAS PROVINCIAS EN FUNCIÓN DEL PORCENTAJE DE ACIERTOS OBTENIDOS POR CADA UNA DE ELLAS Y EN FUNCIÓN DE LA PROVINCIA DE ORIGEN DEL ENCUESTADO

\begin{tabular}{|c|c|c|c|c|c|c|c|c|c|c|}
\hline & Total & & Zamora & & Ávila & & Salamanca & & Cáceres & \\
\hline Provincias & Pos. & $\%$ & Pos. & $\%$ & Pos. & $\%$ & Pos. & $\%$ & Pos. & $\%$ \\
\hline Madrid & 1 & 85,6 & 1 & 85,7 & 1 & 93,2 & 1 & 96,7 & 3 & 83,3 \\
\hline Asturias & 2 & 80,2 & 2 & 82,9 & 3 & 86,4 & 3 & 83,3 & 4 & 83,3 \\
\hline Murcia & 3 & 75,4 & 3 & 71,4 & 2 & 90,9 & 2 & 83,3 & 5 & 75,0 \\
\hline Cantabira & 4 & 68,3 & 4 & 62,9 & 13 & 72,7 & 4 & 73,3 & 6 & 75,0 \\
\hline Salamanca & 5 & 64,7 & 5 & 62,9 & 5 & 81,8 & 7 & 56,7 & 7 & 75,0 \\
\hline Baleares & 6 & 62,3 & 6 & 62,9 & 16 & 70,5 & 5 & 73,3 & 32 & 41,7 \\
\hline Badajoz & 7 & 60,5 & 11 & 42,9 & 7 & 81,8 & 9 & 56,7 & 1 & 91,7 \\
\hline León & 8 & 60,5 & 9 & 48,6 & 6 & 81,8 & 8 & 56,7 & 12 & 58,3 \\
\hline La Rioja & 9 & 59,9 & 14 & 40,0 & 11 & 77,3 & 10 & 56,7 & 8 & 75,0 \\
\hline Zamora & 10 & 59,9 & 7 & 60,0 & 17 & 70,5 & 11 & 56,7 & 9 & 75,0 \\
\hline Cáceres & 11 & 57,5 & 13 & 40,0 & 12 & 75,0 & 14 & 53,3 & 2 & 91,7 \\
\hline Ávila & 12 & 56,3 & 19 & 28,6 & 4 & 84,1 & 6 & 56,7 & 10 & 66,7 \\
\hline Burgos & 13 & 55,7 & 12 & 42,9 & 10 & 77,3 & 13 & 53,3 & 23 & 50,0 \\
\hline Valladolid & 14 & 55,7 & 8 & 51,4 & 9 & 77,3 & 12 & 53,3 & 22 & 50,0 \\
\hline Segovia & 15 & 55,1 & 15 & 40,0 & 8 & 79,5 & 16 & 50,0 & 14 & 58,3 \\
\hline Soria & 16 & 53,9 & 10 & 45,7 & 14 & 72,7 & 17 & 50,0 & 24 & 50,0 \\
\hline Palencia & 17 & 50,9 & 16 & 40,0 & 19 & 68,2 & 15 & 53,3 & 13 & 58,3 \\
\hline Valencia & 18 & 47,3 & 17 & 34,3 & 21 & 59,1 & 18 & 46,7 & 25 & 50,0 \\
\hline Cádiz & 19 & 46,1 & 20 & 28,6 & 15 & 72,7 & 24 & 33,3 & 11 & 66,7 \\
\hline A Coruña & 20 & 43,7 & 18 & 31,4 & 27 & 54,5 & 20 & 40,0 & 15 & 58,3 \\
\hline Sevilla & 21 & 41,3 & 21 & 28,6 & 18 & 70,5 & 26 & 30,0 & 36 & 41,7 \\
\hline Alicante & 22 & 39,5 & 33 & 17,1 & 20 & 61,4 & 21 & 36,7 & 34 & 41,7 \\
\hline Navarra & 23 & 39,5 & 34 & 17,1 & 39 & 45,5 & 19 & 46,7 & 33 & 41,7 \\
\hline Castellón & 24 & 38,9 & 28 & 20,0 & 25 & 56,8 & 22 & 36,7 & 38 & 33,3 \\
\hline Ourense & 25 & 38,3 & 26 & 20,0 & 33 & 50,0 & 23 & 36,7 & 35 & 41,7 \\
\hline Lugo & 26 & 37,1 & 23 & 22,9 & 41 & 43,2 & 25 & 33,3 & 26 & 50,0 \\
\hline Almería & 27 & 36,5 & 37 & 11,4 & 22 & 59,1 & 30 & 23,3 & 17 & 58,3 \\
\hline Pontevedra & 28 & 36,5 & 32 & 17,1 & 34 & 50,0 & 27 & 26,7 & 27 & 50,0 \\
\hline Barcelona & 29 & 35,3 & 22 & 28,6 & 23 & 56,8 & 31 & 23,3 & 41 & 25,0 \\
\hline Córdoba & 30 & 34,7 & 30 & 17,1 & 26 & 56,8 & 32 & 23,3 & 18 & 58,3 \\
\hline Zaragoza & 31 & 33,5 & 25 & 20,0 & 24 & 56,8 & 36 & 20,0 & 29 & 50,0 \\
\hline Toledo & 32 & 32,3 & 31 & 17,1 & 38 & 45,5 & 35 & 23,3 & 19 & 58,3 \\
\hline Melilla & 33 & 32,3 & 24 & 22,9 & 28 & 54,5 & 33 & 23,3 & 42 & 25,0 \\
\hline Ceuta & 34 & 31,7 & 29 & 20,0 & 29 & 54,5 & 34 & 23,3 & 43 & 25,0 \\
\hline Málaga & 35 & 31,1 & 39 & 11,4 & 30 & 54,5 & 50 & 10,0 & 31 & 50,0 \\
\hline Teruel & 36 & 31,1 & 35 & 14,3 & 36 & 47,7 & 28 & 26,7 & 28 & 50,0 \\
\hline Huelva & 37 & 30,5 & 27 & 20,0 & 35 & 47,7 & 37 & 20,0 & 37 & 41,7 \\
\hline Albacete & 38 & 29,9 & 38 & 11,4 & 40 & 45,5 & 29 & 26,7 & 16 & 58,3 \\
\hline Jaén & 39 & 29,9 & 41 & 8,6 & 32 & 52,3 & 44 & 13,3 & 21 & 58,3 \\
\hline Ciudad Real & 40 & 26,3 & 49 & 2,9 & 47 & 34,1 & 39 & 20,0 & 20 & 58,3 \\
\hline Huesca & 41 & 25,7 & 43 & 8,6 & 31 & 54,5 & 41 & 16,7 & 39 & 33,3 \\
\hline Lleida & 42 & 25,7 & 36 & 14,3 & 42 & 43,2 & 45 & 13,3 & 48 & 16,7 \\
\hline Guadalajara & 43 & 24,6 & 44 & 5,7 & 37 & 47,7 & 42 & 16,7 & 40 & 33,3 \\
\hline Granada & 44 & 23,4 & 45 & 5,7 & 43 & 43,2 & 38 & 20,0 & 44 & 25,0 \\
\hline Tarragona & 45 & 23,4 & 40 & 11,4 & 44 & 38,6 & 46 & 13,3 & 52 & 8,3 \\
\hline Cuenca & 46 & 22,2 & 42 & 8,6 & 45 & 34,1 & 43 & 16,7 & 30 & 50,0 \\
\hline Girona & 47 & 19,8 & 46 & 5,7 & 46 & 34,1 & 47 & 13,3 & 45 & 25,0 \\
\hline Álava & 48 & 13,2 & 47 & 5,7 & 49 & 18,2 & 40 & 20,0 & 47 & 16,7 \\
\hline Gipuzkoa & 49 & 13,2 & 52 & 0,0 & 48 & 25,0 & 48 & 13,3 & 49 & 16,7 \\
\hline Bizkaia & 50 & 12,0 & 48 & 5,7 & 50 & 18,2 & 49 & 13,3 & 50 & 16,7 \\
\hline Las Palmas & 51 & 7,2 & 50 & 2,9 & 52 & 6,8 & 51 & 6,7 & 51 & 16,7 \\
\hline Santa Cruz de Tenerife & 52 & 7,2 & 51 & 0,0 & 51 & 9,1 & 52 & 3,3 & 46 & 25,0 \\
\hline
\end{tabular}

Elaboración propia. 
un mayor porcentaje de ocasiones, en el caso de los encuestados con origen en las tres mencionadas provincias de Castilla y León, fueron Madrid, Asturias y Murcia, mientras que en el caso de los cacereños se situaban por delante de estas tres las dos provincias extremeñas con un $91,7 \%$ de aciertos en ambas.

Con la información empleada para elaborar la Figura 2 se incluyó, en la Tabla 4, información sobre la posición que ocupaba cada provincia dentro del ranking de porcentaje de acierto, pero esta vez atendiendo al origen de los estudiantes encuestados. Dicha tabla sirve para contrastar la información de la Figura 2, ya que existe una notable disparidad en las puntuaciones registradas entre los estudiantes originarios de Ávila (56,6\% de aciertos por provincia) y, por ejemplo, los originarios de Zamora (27,4\% de aciertos). EI ranking de las provincias mejor situadas según la procedencia de los estudiantes nos muestra que en los dos primeros cuartiles se sitúan las provincias integradas en Castilla y León y en Extremadura, además de las provincias que se corresponden con las comunidades autónomas uniprovinciales ya mencionadas de Madrid, Asturias, Murcia y La Rioja (ver Tabla 4). En sentido contrario, en el último cuartil del ranking aparecen las dos provincias canarias y las tres vascas, además de Girona. Es destacable que para los alumnos de Ávila, Zamora y Salamanca las provincias castellanomanchegas aparezcan distribuidas entre el tercer y el cuarto cuartil, mientras que, en el caso de los originarios de Cáceres, sitúan mejor el conjunto de las provincias de Castilla-La Mancha, ocupando tres de ellas posiciones dentro del segundo cuartil. Por otra parte, es significativo que las provincias catalanas aparezcan situadas también entre el tercer y cuarto cuartil. No obstante, sobresale siempre Barcelona, que es más conocida que las otras tres, oscilando entre el segundo y el tercer cuartil en función del origen

FIGURA 2

MAPAS MENTALES DE LOS ALUMNOS ORIGINARIOS DE LAS DISTINTAS PROVINCIAS ANALIZADAS

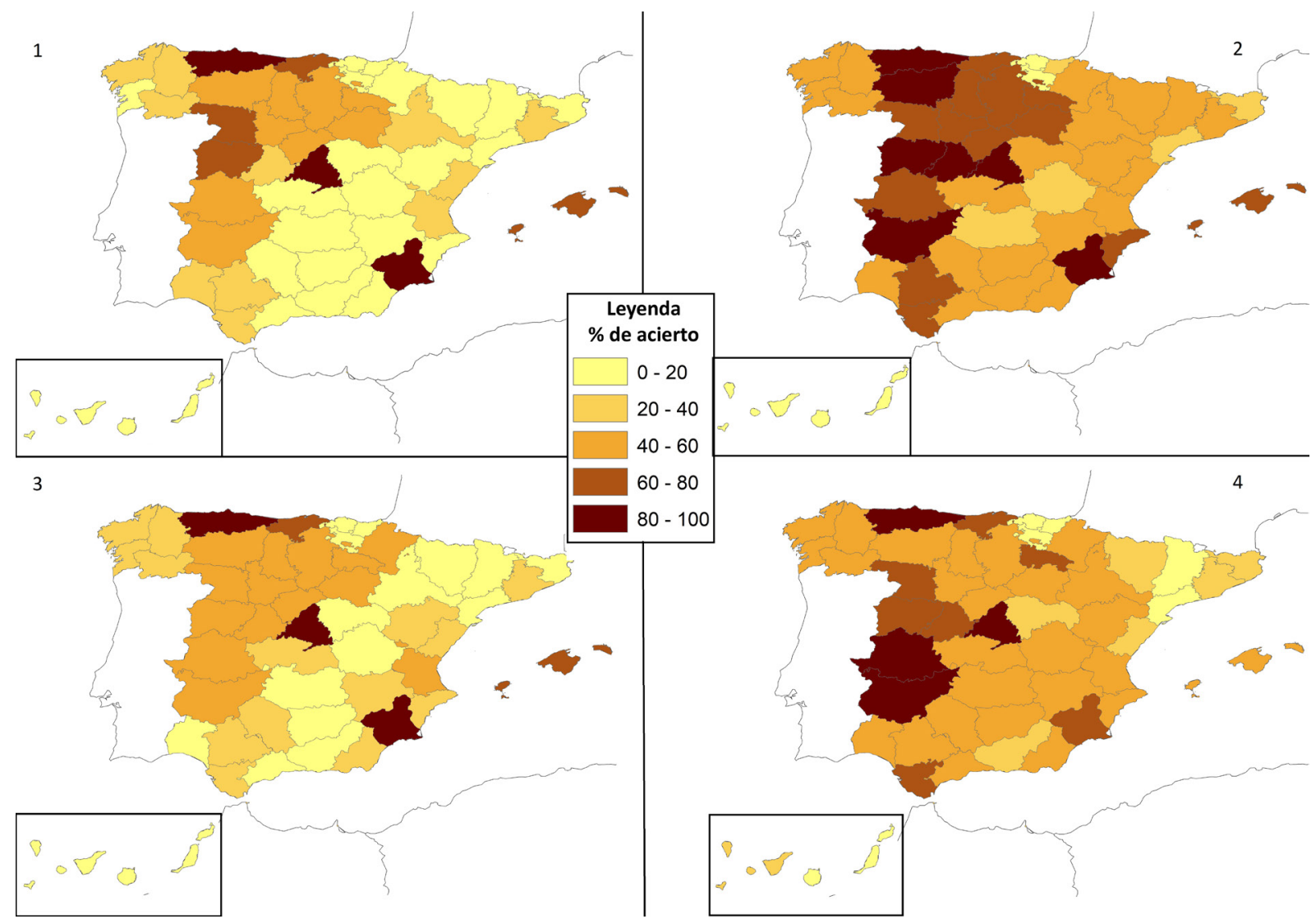

Elaboración propia. Nota: 1) mapa mental de los alumnos originarios de Zamora ( $n=35) ; 2)$ mapa mental de los alumnos originarios de Ávila ( $n=44) ; 3$ ) mapa mental de los alumnos originarios de Salamanca $(n=30)$; 4) mapa mental de los alumnos originarios de Cáceres $(n=12)$. 
de los encuestados. Por tanto, las peor conocidas por los encuestados son territorios alejados de la provincia de origen, mientras que el mayor conocimiento coincide con las unidades próximas al lugar de residencia del alumnado participante. También, siendo una excepción a este patrón, obtienen gran valoración las provincias que, a su vez, se constituyen en comunidades autónomas uniprovinciales.

Desde otro punto de vista, se manifiesta una sutil diferencia en los resultados entre los estudiantes procedentes de Ávila, Salamanca y Zamora y el grupo de estudiantes originarios de Cáceres, ya que, en el caso de estos últimos, las dos provincias extremeñas aparecen en los primeros lugares del ranking y el grado de conocimiento de las provincias castellanomanchegas es notablemente superior a la de los alumnos originarios de las tres provincias de Castilla y León analizadas. Esto puede deberse a que las citadas provincias castellanoleonesas son provincias cercanas y pertenecientes a una misma comunidad autónoma, mientras que los alumnos extremeños vienen de otra comunidad autónoma con vivencias diferentes. Por ello, sería interesante ampliar este tipo de análisis a un alumnado más ecléctico en su procedencia, también más numeroso. Con ello se conseguiría tener una perspectiva más amplia y certera de los conocimientos sobre el mapa de España entre los futuros maestros.

\section{CONCLUSIONES Y DISCUSIÓN}

Este trabajo nos ha permitido realizar una primera aproximación a los conocimientos sobre la organización territorial de España que poseen un grupo de estudiantes del grado en Maestro en Educación Primaria, obteniendo los siguientes resultados: a) un tercio de los futuros docentes no fue capaz de ubicar de forma correcta, al menos, 10 provincias; $b$ ) más de la mitad de los encuestados no fue capaz de situar adecuadamente 25 provincias. En consecuencia, el grado de alfabetización sobre la organización políticoadministrativa de España de los encuestados es muy deficiente, algo que también han demostrado otros estudios sobre conocimientos mínimos en geografía de docentes en formación de Educación Primaria (Binimelis y Ordinas, 2016). Los resultados obtenidos parecen corroborar algunas de las conclusiones obtenidas por Binimelis y Ordinas (2018) en su análisis de los mapas mentales sobre las Islas Baleares realizados por alumnos de magisterio de la misma comunidad. Desde esta óptica, suscribimos que "en los conocimientos geográficos de los universitarios el peso de la experiencia vital es mucho mayor que el de la experiencia académica" (p. 350), algo que también ha sido identificado por otros investigadores y en otros territorios, como señalaron Rédei, Kincses y Jakobi (2011) en su investigación llevada a cabo en Hungría.

Por otra parte, los resultados muestran una diferencia de género, mostrando las mujeres una puntuación significativamente más alta que la de sus compañeros varones, siendo este un contrapunto a la mayoría de las tesis y afirmaciones realizadas sobre este aspecto (Lawton, 2018). Además, se ha observado una relación positiva entre los resultados obtenidos y la formación de los progenitores, algo que refuerza las conclusiones de otros trabajos de otras áreas de conocimiento en las que se señala la importancia del nivel académico de los padres en el rendimiento académico de los alumnos (Garbanzo, 2007; Hernando, Oliva y Pertegal, 2012). El propio informe PISA (OECD, 2019) reconoce la importancia del contexto social a la hora de que los alumnos adquieran determinadas competencias, señalando expresamente, como uno de los factores determinantes, el nivel cultural de las familias.

En cuanto al instrumento utilizado, el mapa mental, parece demostrar, de nuevo, su utilidad para llevar a cabo un diagnóstico, en forma de investigación sobre conocimientos mínimos de geografía, algo que también señalaron algunos trabajos (Polonsky, Novotny y Lysak, 2010). A través de él se ha podido evaluar el conocimiento que el alumnado participante tiene sobre el mapa provincial de nuestro país. No obstante, el recurso ofrece muchas más posibilidades que no hemos podido encarar en este primer análisis. El perfil y la forma del mapa, la adecuación del tamaño de España a su tamaño real son aspectos de la geometría de los esbozos que abrirían nuevos retos en la investigación.

Este trabajo presenta una serie de limitaciones que conviene mencionar. En primer lugar, la muestra analizada se circunscribe a un territorio concreto de nuestro país, por lo que será necesario ampliar el estudio a otras universidades para enriquecer los resultados. En segundo lugar, no se ha analizado la corrección ortográfica en la es- 
critura de los topónimos provinciales, lo que podría haber permitido evaluar otras competencias vinculadas con las ciencias sociales. Y tampoco se ha evaluado a los estudiantes al terminar el grado de Maestro en Educación Primaria, con lo que se podría haber analizado lo que han aprendido sobre este tema a lo largo de su formación universitaria, y demostrar así el grado de utilidad de la misma. Estas cuestiones deben ser tomadas en consideración en futuros estudios con el objetivo de consolidar esta línea de investigación.

Por último, es necesario plantear una reflexión a la luz de los resultados obtenidos. ¿Cómo es posible que las nuevas generaciones de estudiantes que inician el grado en Maestro en Educación Primaria presenten unos conocimientos sobre la organización territorial de España tan poco rigurosos, al menos en lo referido al mapa provincial de nuestro país? ¿En qué está fallando el sistema educativo actual? Es cierto que los alumnos encuestados podrían "refrescar" sus conocimientos sobre el tema a lo largo del grado, pero es realmente llamativo que alumnos que han cursado la formación obligatoria y el bachillerato presenten unos conocimientos limitados sobre la organización territorial de nuestro país. Es evidente que se necesitan realizar más investigaciones para identificar los puntos débiles de este sistema para, a continuación, tratar de aportar soluciones realistas a medio plazo, entre las que se podrían incluir la puesta en práctica de metodologías apoyadas en la gamificación a través de alguno de los juegos en línea existentes, tales como la familia Seterra o GeoSapiens del Instituto Geográfico Nacional, para mejorar el conocimiento de las provincias españolas. Quizás sería necesario plantear una evaluación de conocimientos mínimos relacionados con la geografía en pruebas como el mencionado informe PISA, para obtener un diagnóstico más detallado. No obstante, los resultados obtenidos nos animan a profundizar en una línea de trabajo que puede arrojar más luces sobre los puntos débiles en la formación de los futuros maestros, algo que como profesionales de la educación nos preocupa. Además de un interés científico, los frutos de esta reflexión podrían ayudar a la Administración a replantearse algunas cuestiones sobre las lagunas de nuestro sistema educativo.

\section{REFERENCIAS BIBLIOGRÁFICAS}

Álvarez Garay, B., Córdoba Gamboa, L. (2015). La geoalfabetización en la Universidad Estatal a Distancia: trabajo con funcionarios y estudiantes. En G., Buzai (Ed.), XV Conferencia Iberoamericana de Sistemas de información Geográfica (pp. 16-23). Valparaíso, Chile: Pontificia Universidad Católica de Valparaíso.

Archila, P. A.; Molina, J. y Truscott de Mejía, A. (2019). Promoting undergraduates' awareness of the importance of thinking critically about false or inaccurate scientific information presented in news articles. Revista Eureka sobre Enseñanza y Divulgación de las Ciencias, 16(3), 3106-1 3106-27. https://doi.org/10.25267/Rev_Eureka_ ensen_divulg_cienc.2019.v16.i3.3106

Badillo Vargas, C. A., Crespo Allende, N. y Hass Prieto, V. (2017). Alfabetizando en geografía: medios y modos para educar la mirada de quienes comienzan en la comprensión de esta disciplina. Educación: revista de la Universidad de Costa Rica, 41(2), 1-15. http://dx.doi.org/10.15517/revedu. v41i2.17953

Bascom, J. (2011). Geographic Literacy and Moral Formation among University Students. Review of International Geographical Education Online, 1(2), 92-112.

Binimelis Sebastián, J., García De La Vega, A., García González, J. A., Gómez Gonçalves, A., Gómez Trigueros, I., Jeréz García, Ó., Ordinas, A., Rodríguez Domènech, M. A., Rodríguez, M., Serrano, Ó. y Varela Ona, R. (2018). Alfabetización en Geografía y mapas mentales. Nacimiento de un proyecto inter-universitario de Educación y Geografía de la percepción. En A. García de la Vega (Ed.), Contribución didáctica al aprendizaje de la geografía (pp. 429-439). Madrid, España: Universidad Autónoma de Madrid y Asociación Española de Geografía.

Binimelis Sebastián, J. y Ordinas Garau, A. (2016) Los conocimientos mínimos de Geografía en los estudios de Grado en Educación Primaria. Revista complutense de educación, 27(3), 1309-1326. https:// doi.org/10.5209/rev_RCED.2016.v27.n3.48586

Binimelis Sebastián, J. y Ordinas Garau, A. (2018). Alfabetización en Geografía y mapas mentales. Los conocimientos mínimos entre los estudiantes universitarios de Educación Primaria. Cuadernos geográficos de la Universidad de Granada, 57, 330-351. http://dx.doi.org/10.30827/cuadgeo. v57i1.5528 
Burgueño, J. (2011). La invención de las provincias. Madrid, España: La Catarata.

Butt, G., Weeden, P. y Wood, P. (2004). Boys' Underachievement in Geography: An Issue of Ability, Attitude or Assessment? International Research in Geographical and Environmental Education, 13(4), 329-347. https://doi. org/10.1080/14724040408668454

Cachinho, H. y Reis, J. (2011). Educación geográfica y ciudadanía en Portugal: de los discursos a la práctica en los centros escolares. Didáctica Geográfica, 9, 185-204.

Cavagnetto, A. R. (2010). Argument to Foster Scientific Literacy: A Review of Argument Interventions in K-12 Science Contexts. Review of Educational Research, 80(3), 336-371. https://doi. org/10.3102/0034654310376953

Clark, A.M., Monk, J. y Yool, S.R. (2007). GIS Pedagogy, Web-based Learning and Student Achievement. Journal of Geography in Higher Education, 31(22), 225-239. https://doi. org/10.1080/03098260601063677

Claudino, S. (2010). Portugal en los libros de texto españoles de Geografía. En M. J. Morrón (coord.), Geografía, educación y formación del profesorado en el marco del espacio europeo de educación superior (pp. 175190). Murcia, España: Grupo de Didáctica de la Geografía de la Asociación de Geógrafos Españoles.

Clemente Díaz, M. (1992). Psicología Social. Métodos y técnicas de investigación. Madrid: Eudema.

Corrochano, D., Gómez-Gonçalves, A., SánchezBarbero, B. y Martín-Pastor, E. (2019). Field trips and other teaching resources in natural and social sciences: educational implications from past experiences in Spanish primary schools. History of Education and Children's Literature, XIV(1), 779798. https://doi.org/10.1400/269814

DECRETO 26/2016, de 21 de julio, por el que se establece el currículo y se regula la implantación, evaluación y desarrollo de la Educación Primaria en la Comunidad de Castilla y León (BOCyL núm. 142, de 25 de julio de 2016, 34184-34283).

De Castro Aguirre, C. (1999). Mapas cognitivos. Qué son y cómo explorarlos. Scripta Nova. Revista electrónica de Geografía y Ciencias Sociales, 33(1). Recuperado de http://www.ub.edu/geocrit/sn-33.htm

Díaz Moreno, N. (2019). Caracterizando controversias sociocientíficas en la prensa escrita. Una he- rramienta para el desarrollo de la alfabetización científica. Revista Eureka sobre Enseñanza y Divulgación de las Ciencias, 16(1), 1102-1 1102-13. https://doi.org/10.25267/Rev_Eureka_ensen_divulg_cienc.2019.v16.i1.1102

Garbanzo Vargas, G. M. (2007). Factores asociados al rendimiento académico en estudiantes universitarios, una reflexión desde la calidad de la educación superior pública. Revista Educación, 31(1), 43-63.

García González, J. A. (2018). Análisis multitemporal en ciudades medias con mapas de Albacete: 19932016. Cuadernos Geográficos, 57(1), 197-218. http://dx.doi.org/10.30827/cuadgeo.v57i1.5755

Gersmehl, P. (2005). Teaching Geography. Nueva York, Estados Unidos: Guilford Press.

Gersmehl, P.J. y Gersmehl, C.A. (2011). Spatial Thinking: Where Pedagogy Meets Neurocience. Problems of Education in the 21st Century, 27, 48-66.

Gold, J. R. (2009). Behavioral Geography. En R. Kitchin y N. Thrift (Eds.), International Encyclopedia of Human Geography (pp. 282-293). Elsevier.

Hernando, Á., Oliva, A. y Pertegal, M. A. (2012). Variables familiares y rendimiento académico en la adolescencia. Estudios de Psicología, 33(1), 51-65. https://doi.org/10.1174/021093912799803791

Ikhsan, F. A., Kurnianto, F. A., Apriyanto, B. y Nurdin, E. A. (2018). Geography skills domain taxonomy. Geosfera Indonesia, 2(1), 54-59. https://doi. org/10.19184/geosi.v2i1.7525

Kolossov, V. (2016). Declaración Internacional Sobre Educación Geográfica. Didáctica Geográfica, 17, 257-274.

Lawton, C.A. (2018). Sex and gender in geographic behavior and cognition. En D. R. Montello (Ed.), Handbook of Behavioral and Cognitive Geography (pp. 247-259). Glocester, Reino Unido: Edward Elgar Publishing.

Lee, J. y Bednarz, R. (2012). Components of Spatial Thinking: Evidence from a Spatial Thinking Ability Test. Journal of Geography, 111(1), 15-26. https:// doi.org/10.1080/00221341.2011.583262

Lynch, K. (1960). La imagen de la ciudad. Barcelona, España: Editorial Gili.

Martín Lou, M. A., Lozano de San Cleto, M. J. y Lázaro y Torres, M. L. (2006). ¿Conoces España? Resultados de un concurso escolar. Boletín de la R. S. G., CXLII, 375-453. 
Memisoglu, H. (2017). Opinions of Teachers and Preservice Teachers of Social Studies on Geo-Literacy. Educational Research and Reviews, 12(19), 967979. https://doi.org/10.5897/ERR2017.3315

Morales Yago, F. J. (2015). La ciudad de Valencia como espacio percibido por los estudiantes universitarios. Aportaciones desde el análisis propuesto por la geografía de la percepción. Estudios Geográficos, 76(278), 203-233. https://doi.org/10.3989/ estgeogr.201507

Morales, F. J., de Lázaro, M. L. y Gómez, M. L. (2018). University students' perceptions of the inner cities of Murcia and Valencia. Quaestiones Geographicae, 37(3), 75-85. https://doi.org/10.2478/ quageo-2018-0026

Nishimoto, S. (2012). Evaluating Mental Maps. University of Oregon, (unpublished master thesis).

Nolan, R. E. (2002). Geo-literacy: how well adults understand the world in which they live. Adult Basic Education, 12(3), 134-144.

OECD (2019). PISA 2018 Results (Volume I): What Students Know and Can Do. https://doi. org/10.1787/5f07c754-en

ORDEN EDU/362/2015, de 4 de mayo, por la que se establece el currículo y se regula la implantación, evaluación y desarrollo de la educación secundaria obligatoria en la Comunidad de Castilla y León (BOCYL núm. 86, de 8 de mayo de 2015, 32051-32480).

Pérez Sánchez, A. M. y Castejón Costa, J. L. (1998). Un modelo causal-explicativo sobre la influencia de las variables psicosociales en el rendimiento académico. Bordón. Revista de pedagogía, 50(2), 171-185.

Polonsky, F., Novotny, J. y Lysak, J. (2010). Cognitive Mapping of Major World Regions among Czech Geography Students. Journal of Maps, 6(1), 311318. https://doi.org/10.4113/jom.2010.1083

Puyol, R. (1980). Región y comarca. En A.G.E. (ed.). La Región y la Geografía española (pp. 79-89). Valladolid, España: Asociación de Geógrafos Españoles.

Redei, M., Kincses, Á. y Jakobi, Á. (2011). The World seen by Hungarian students: a mental map analysis. Hungarian Geographical Bulletin, 60(2), 135-159.

Roberts, D. A. y Bybee, R. W. (2014). Scientific Literacy, Science Literacy, and Science Education. En N. G. Ledermna; S. K. Abell (Eds.), Handbook of Research on Science Education (pp. 545-558). Nueva York, Estados Unidos: Routledge.
Rubio García, S. y Martínez Medina, R. (2014). ¿Es competente el alumnado del Grado de Educación Primaria en la comprensión del tiempo atmosférico y el clima? En R, Martínez; E. M. Tonda (Eds.), Nuevas perspectivas conceptuales y metodológicas para la educación geográfica (133-151). Murcia, España: Grupo de Didáctica de la Geografía de la Asociación de Geógrafos Españoles.

Rudolph, J. L.; Horibe, S. (2015). What do we mean by science education for civic engagement? Journal of Research in Science Teaching, 53(6), 805-820. https://doi.org/10.1002/tea.21303

Saarinen, T. F. (1987). Centering of mental maps of the world. Tucson, Estados Unidos: Department of Geography and Regional Studies. University of Arizona.

Saarinen, T. F.; MacCabe, C. L. (1995). World patterns of geographic literacy based on sketch map quality. The professional geographer, 47(2), 196-204. https://doi.org/10.1111/j.00330124.1995.00196.x

Santiago Rivera, J. A. (2017). La alfabetización geográfica comunitaria desde la práctica escolar cotidiana de la geografía escolar. Revista brasileira de Educação em geografia, 7(14), 24-43. https://doi. org/10.46789/edugeo.v7i14.528

Smith, F. (2004) "It's not all about grades": Accounting for gendered degree results in geography at Brunel University, Journal of Geography in Higher Education, 28(2), 167-178. https://doi. org/10.1080/0309826042000242422

Solís Trapero, E. y Ureña Francés, J. M. (2019). La ciudad como espacio percibido. El caso de Toledo. Toledo, España: UCLM. Cuadernos de Ingeniería, Arquitectura y Territorio.

Souto, X. M. (2008). Una educación geográfica para el siglo XXI: aprender competencias para ser ciudadano en el mundo global. Revista virtual. Geografía, Cultura y Educación, 1, 28-47.

Sudas, I. y Gokten, C. (2012). Cognitive maps of Europe: geographical knowledge of Turkish geography students. European Journal of Geography, 3(1), 41-56.

Turner, S.; Leydon, J. (2012). Improving Geographic Literacy among First-Year Undergraduate Students: Testing the Effectiveness of Online Quizzes. Journal of Geography, 111(2), 54-66. https://doi. org/10.1080/00221341.2011.583263 